\title{
APPLICATION OF FUZZY-MRP-II IN FAST MOVING CONSUMER GOODS MANUFACTURING INDUSTRY
}

\author{
Jiping Niu \\ John Dartnall \\ Faculty of Engineering \\ University of Technology, Sydney \\ 15 Broadway, Ultimo, NSW, 2007, AUSTRALIA
}

\begin{abstract}
In today's global marketplace, the information associated with a product is fast becoming a critical link in the supply chain. Especially in fast moving consumer goods (FMCGs) industry, there is more fierce competition and more unstable requirement. Most plants use Manufacturing Resource Planning (MRP-II) system to manage the process of production. But how to deal with the uncertain and imprecise requirement in the middle term production schedule and make a best plan for best profit is a main problem in this system. In this paper, we investigate the method named Fuzzy-MRP-II to deal with the uncertainty and imprecision. Fuzzy-MRP-II shows all of the information for the decision makers allowing them to consider all possibilities of the orders.
\end{abstract}

\section{INTRODUCTION}

Nowadays, market competition is fierce, especially in fast moving consumer goods industry. Specific properties of fast moving consumer goods industry lead to forecast requirement becoming more important. Firstly, in a fast moving customer goods company, the products are diversified. At the same time it means the inventory of raw materials and products carry a lot of cost. Secondly, consumers have many choices in the market; the level of customer satisfaction becomes a very important Key Performance Index (KPI) for a company. Delivering on time, correct demand and good quality are the main parameters for this KPI. It becomes an important element for the company's existence.

How to avoid shortage of goods in the market and decrease or keep the minimum level of inventory and optimize using production capability as well become the most important key issues in fast moving consumer goods industry. Unfortunately, company always can not wait for confirmed orders from their consumers. They still need time to prepare all of the resources for production.
Based on this aim, how to make a correct mid-term production schedule when the decision maker faces the uncertain and imprecise requirement information becomes a very interesting point.

There are three major ways for dealing with uncertainty used in manufacturing (Grabot et al. 2005):

- Safety margins in the evaluation of quantities and delays,

- Periodic refresh of the various production plans (rolling horizon plans) with time fences in order to freeze the production plan at a given time horizon in order to limit short term modifications,

- Estimation of safety inventory levels to face unexpected demand increase or delivery problems (generally achieved according to probabilistic approaches).

These techniques generally require statistical and probabilistic knowledge on the behaviour of the production system and of its providers and customers. However, when the products become diversified, the above methods may not be suitable (Orlicky and Plossl 1994). And also, if the decision maker is less experience, he can not make a correct plan. There is a method called Fuzzy-MRP-II supply a way to solve the problem.

There are two main contributions of Fuzzy-MRP-II. Firstly, the produced information is richer semantically than the information used in a traditional MRP approach which is only an advantage if the information is made understandable to a user non-specialist of the possibility theory. Secondly, MRP-II engines are often in the core of the management and information exchange on the orders within SCM. The Fuzzy set can be integrated in a homogeneous way within this approach since the produced outputs of an F-MRP system can be taken as an input by another F-MRP system. 
2

FUZZY MODELLING OF ORDERS

In this context, the modelling progress following the steps (Efstathiou 1990) (Figure 1):

List the input variables that affect the decision of production plan:

- Determine the fuzzy membership values associated with the input variables

- Determine the rules, which are to be fired in the rule base

- Use FIS(Fuzzy Inference System) to determine appropriate output fuzzy

- Choose method to determine the crisp value for the output variable

- Defizzifier for the result
- Available production capacity operating factor

- Inventory cost rate

- Company's special strategies

The second step is to define the inputs' possibility distribution membership functions and the weight of each one. In fuzzy set theory, trapezoidal functions were recommend as easily be applied on the obtained quantities.

In the end, the boundaries of each input levels are base on the membership functions, such as low, medium, high different levels.

Starting from the value of the gross requirement $\mathrm{GR}(\mathrm{P}, \mathrm{T})$ and taking into account the inventory level of product $\mathrm{P}$ at Period $\mathrm{T}, \operatorname{Inv}(\mathrm{P}, \mathrm{T})$, the net requirement.

$$
N R(P, T)=G R(P, T) \Theta \operatorname{Inv}(P, T)
$$

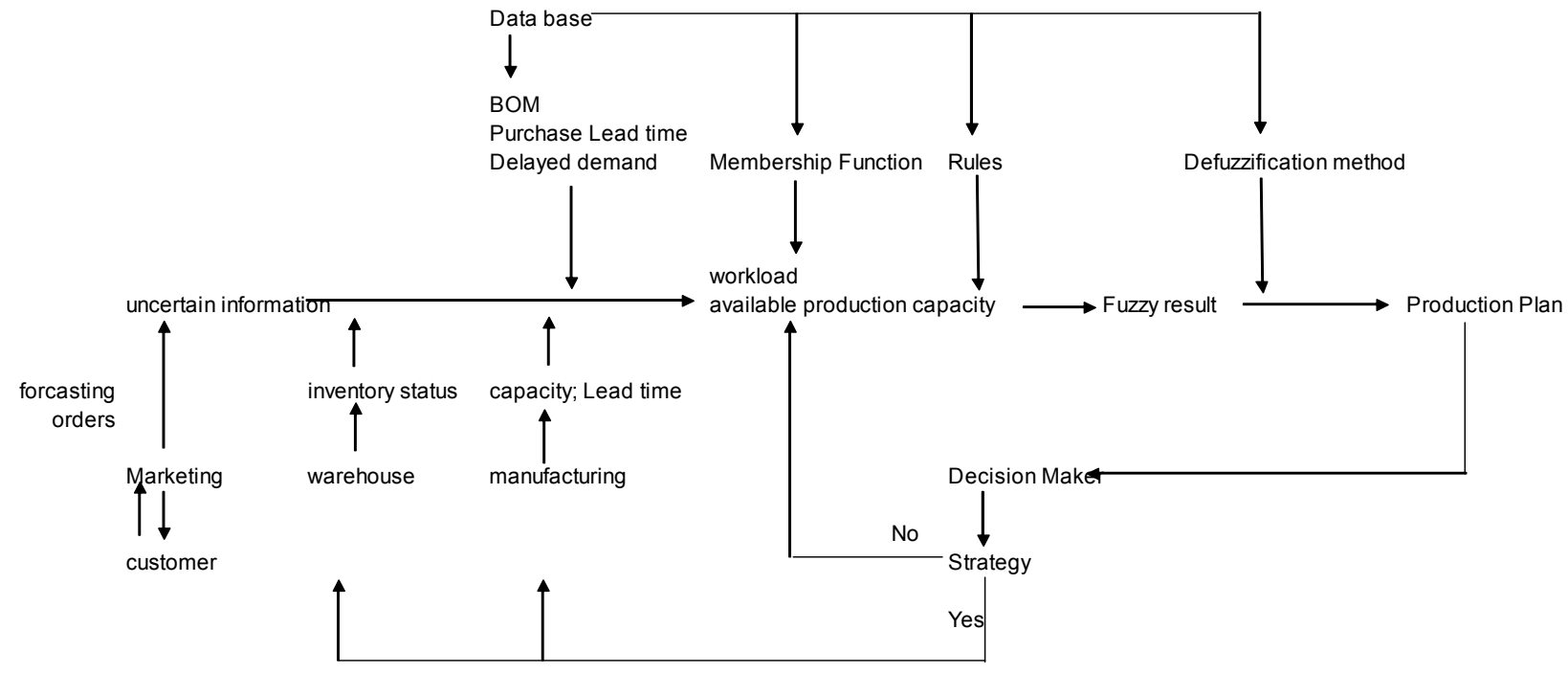

Figure 1: Process of Fuzzy-MRP

In this paper, we use Matlab software to apply the method. Matlab is a menu driven software that allows the implementation of fuzzy constructs like membership functions and database of decision rules. The software is easy to use and it is user friendly. The face of the software showed in Figure 2.

\subsection{Fuzzification of the Input}

Firstly, it is important to define which factors are effective on the result as profit of production plan as the fuzzy set's inputs:

Normally, in FMCG industry, there are four main elements have to be considered when make a mid-term production plan:

- Net requirement workload
The following calculations will be used as the basic operations on trapezoidal fuzzy set. Because it is easy to be apply. They were defined in Dubois and Prade (1989).

$$
\begin{gathered}
A_{i} \oplus A_{j}=(a, b, c, d, h) \text { with } h=\min \left(h_{i}, h_{j}\right) \\
c=h\left(\frac{c_{i}}{h_{i}}+\frac{c_{j}}{h_{j}}\right), \quad d=h \cdot\left(\frac{d_{i}}{h_{i}}+\frac{d_{j}}{h_{j}}\right) \\
A_{i} \Theta A_{j}=(a, b, c, d, h) \quad \text { with } \quad h=\max \left(h_{i}, h_{j}\right) \\
c=h \cdot\left(\frac{c_{i}}{c_{j}}+\frac{d_{j}}{h_{j}}\right) \quad d=h \cdot\left(\frac{d_{i}}{h_{i}}+\frac{c_{j}}{h_{j}}\right)
\end{gathered}
$$




\section{Niu and Dartnall}

\section{AFIS Editor: Untitled}

File Edit View

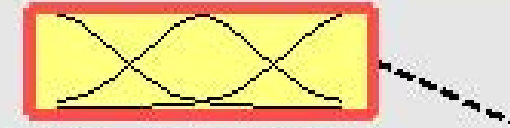

NetRequirementworkload

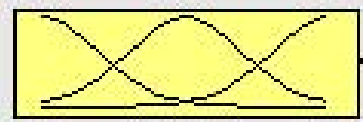

ailableProductionCapacityOperating Factor.

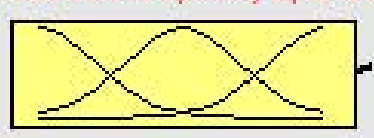

InwentarvicastRate

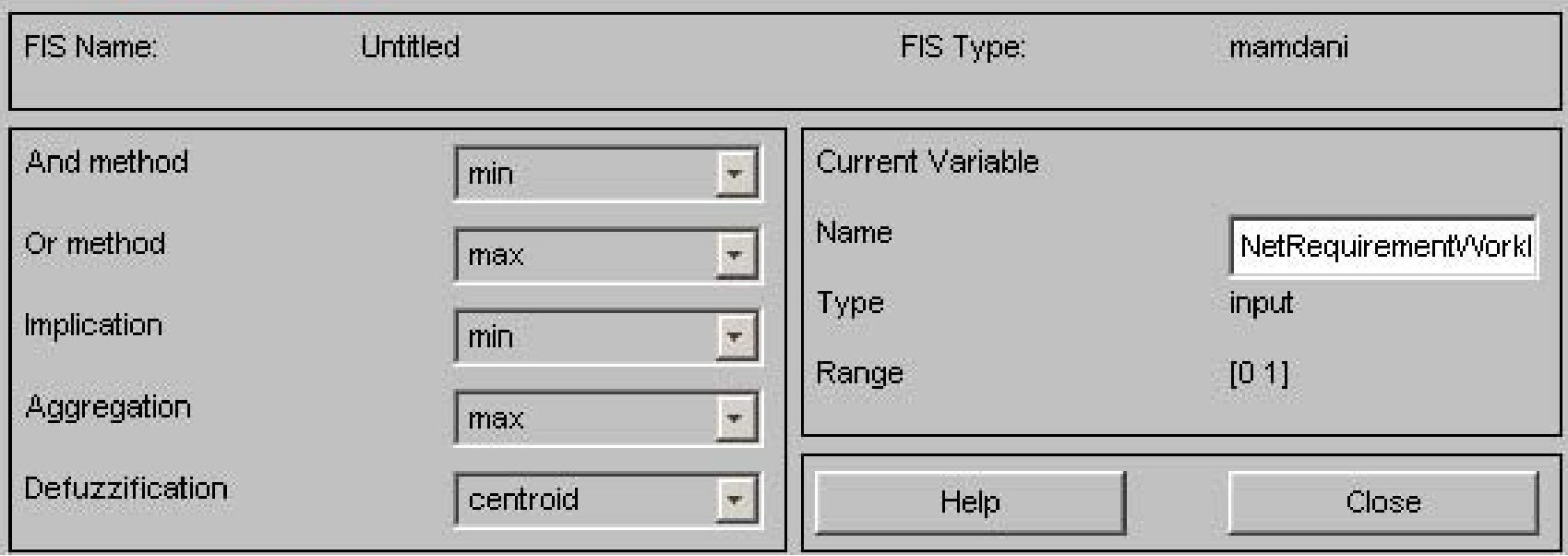

Figure 2: Fuzzy set model in Matlab

An uncertain order can be represented with a union of trapezoids: $A=\left(a, b, c, d, h^{\prime}\right) \cup\left(0,0,0,0, h^{\prime \prime}\right)$

Let's consider an illustrative example:

$$
\begin{aligned}
& N R(P, T)=\quad(120,160,10,20,0.8) \\
& (152,210,10,15,1) \cup(240,260,0,15,0.6)
\end{aligned}
$$

with a product $\mathrm{P}$ being manufactured by lots of 50 parts (Figure.3.).

A workload includes set-up time (time for reconFigureuring the machine during a change of lot) and processing time (of one product on the machine for a manufacturing operation):

$$
\mathrm{WL}(\mathrm{P}, \mathrm{T})=\text { set-up time }{ }^{\oplus} \mathrm{LR}(\mathrm{P}, \mathrm{T})^{*} \text { process time }
$$

Let's suppose that these products are manufactured on the same machine or production line $\mathrm{M}$, with respective set-up times of $10 \mathrm{~min}$ and respective processing time per part of $20 \mathrm{~min}$. The result can be seen in Figure 4.

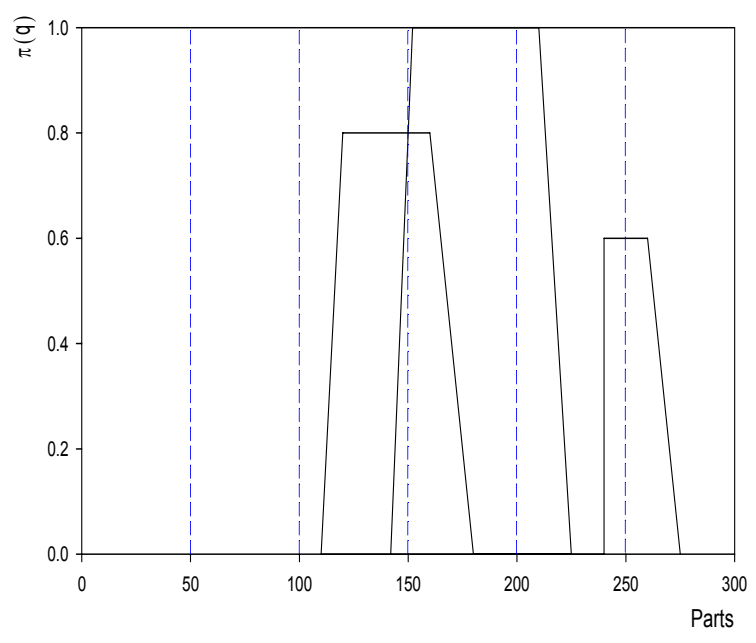

Figure 3: Determination of the number of lots 


\section{Niu and Dartnall}

$W L(M)=10 \oplus(20 *(152,210,10,15,1) \cup$

$(240,260,0,15,0.6) \cup(120,160,10,20,0.8)$

$=(2410,3210,200,400,0.8) \cup(4810,5210$,

$0,300,0.6) \cup(3050,4210,200,300,1)$

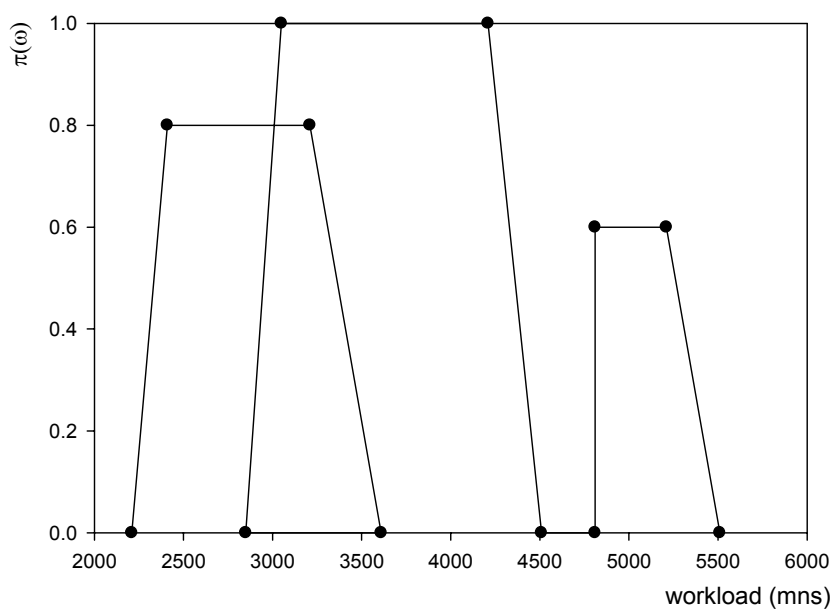

Figure 4: Workload distribution

As a matter of fact, Figure (4) shows the possibility distribution $\pi(w)$ of the workload. Let $\mathrm{S}$ be the successive possible values of the load. We can calculate

$$
\pi(R \geq S)=\sup \pi(w) \text { for } w \geq S
$$

At the same time, the necessity that

$$
q \geq 0, N(q \geq 0)=\inf (1-\pi(q)) \text { for } q \leq 0
$$

This is only the right part of the possibility distribution has been exploited. In order to get more comprehensive information (Grabot et al. 2005)

From Figure 5, we can see three different levels of workload, the first level called fully necessary load, the second one is fully possible load, and the last one is maximum load. This is the first input of FIS (Fuzzy Inference System).

The second input is available production capacit operating factor. We define three different levels: machine idle; normal; overtime.

The third input is Inventory cost rate. We also can define three different levels as different prices in market: low; medium; high.

In different company they can have different strategies which effect on the production plan. Such as some productions related the brand of company, then they become to very important. When decision-maker makes the decision of production plan they will prefer to choose maximum workload.

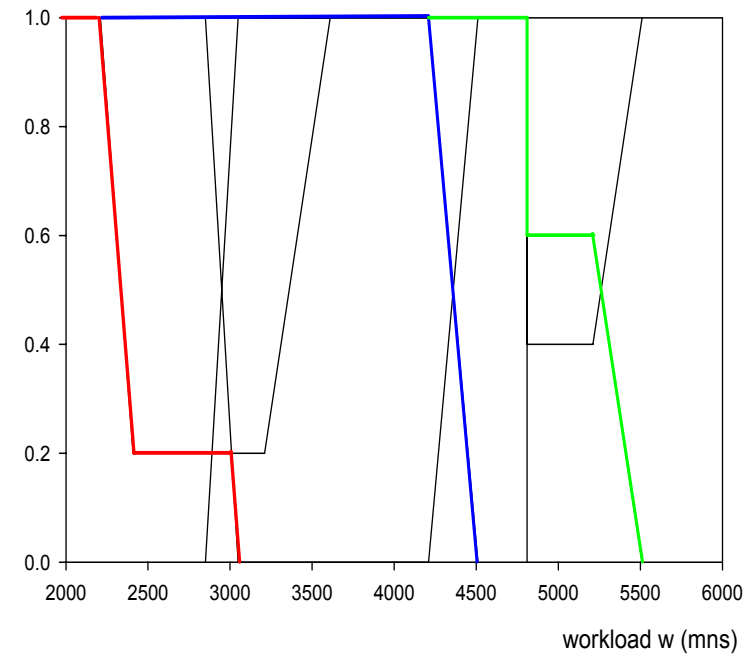

Figure 5: Load planning of $\mathrm{R}$ on delivers time

\subsection{The Fuzzy Reasoning as Inferences}

Inferences are mapping processes from inputs to outputs. In manufacturing industry it includes the company's strategies.

An inference step (the evaluation of a rule) consists of three steps as follow:

- Aggregation: aggregation is the calculation of the fulfillment of the whole rule, base on the fulfillments of the individual premises. This process is the computing of "IF" part, generally corresponds to the logical AND operator of the individual premise expressions.

- Implication: Implication based on the certainty factors of the premises, calculates the corresponding degree of certainty for the conclusion. This is called the degree of fulfillment. This step represents the conclusion of the logic statement "IF... Then..."

- Accumulation: The classical operators for these functions are: $\mathrm{AND}=\min , \mathrm{OR}=\max$, and NOT $=$ additive complement. (Kahraman 2007)

Base on the three inputs in this case, we can give rules to inference system.

For an example in this case "IF the net requirement workload is maximum, AND the available capacity operating factor is low, THEN the profit is very low".

This item of strategy explained as, if the decision maker made a decision as the maximum of workload, and means the company will take the risk of high inventory. The second condition is the available capacity operating 


\section{Niu and Dartnall}

factor is low, which means there is idle capacity. So, the

compare the results between traditional MRP and FuzzyMRP.

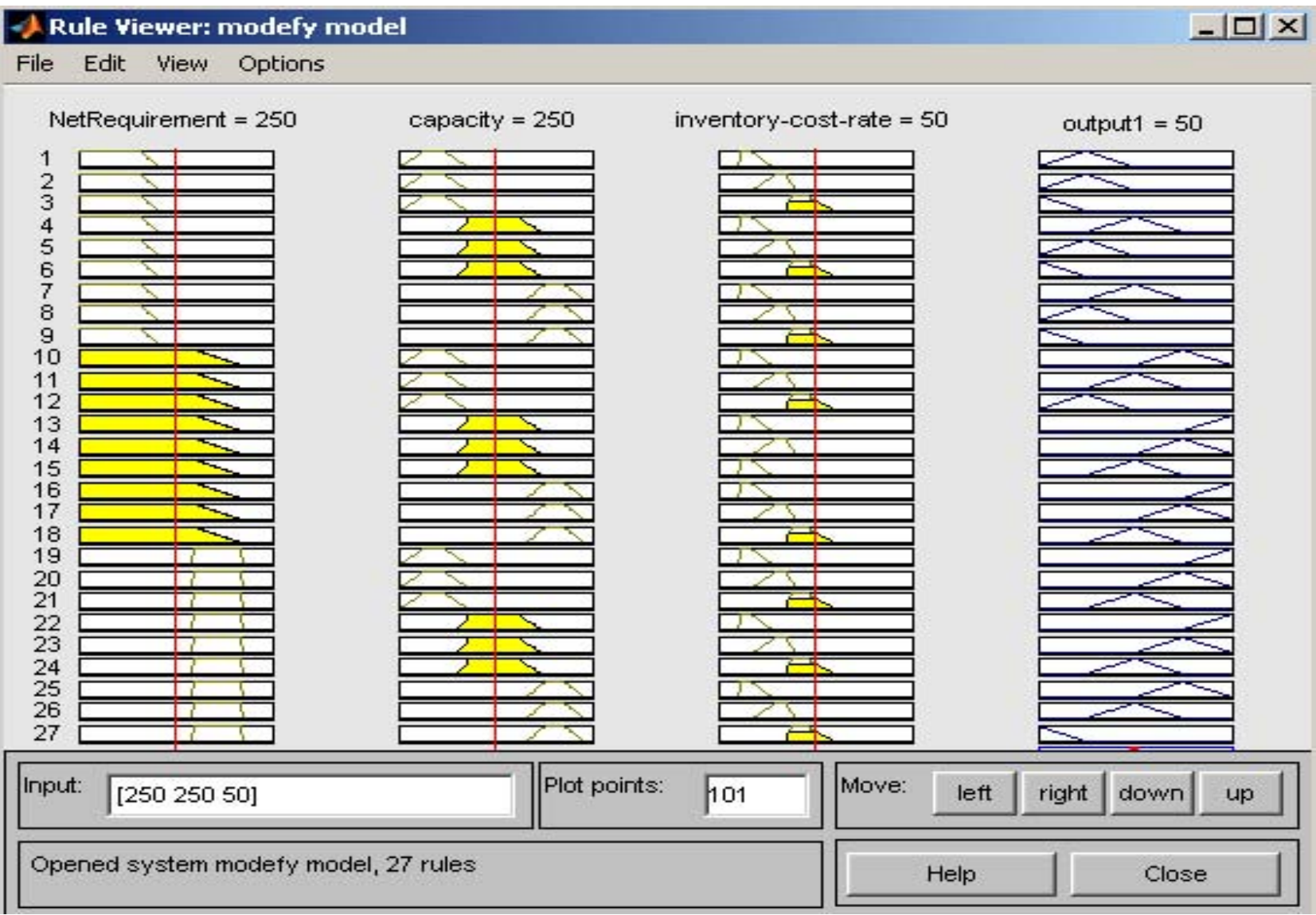

Figure 6: Inference rules in Fuzzy set

company will take two kinds of risks. Then, the profit as result will be defined as very low.

The rules showed as above chart, which is expressed by Matlab Toolbox Fuzzy logic function.

\subsection{Defuzzification as an Output}

Defuzzification in Fuzzy theory means translation of the results of the inference process of a knowledge based system (membership of terms of the linguistic variables) into (crisp) values, or a recommendation of the action to take. (Rondeau et al. 1997) In this case, that will be an idiographic production plan (workload planning) which can get the best profit. In this paper, we just used a very simple example as simulating model; the result can be seen in Figure. 7 and Figure.8.

In this paper, we considered a simulated case to express Fuzzy-MRP model's process. So we didn't give much detail, such as weigh of each input. The further work will be put the model into a real MRP system and

\section{CONCLUSIONS}

This approach of an integrated management of uncertain orders in a MRP process and then get the best profit of production as aim leads us to formulate two conclusions.

Firstly, fuzzy logic allows for set membership values to range (inclusively) between 0 and 1 , and in its linguistic form, imprecise concepts like "poor", "medium", and "good". So, it is easy to be understood by user and applied.

Specifically, in FMCGs manufacturing management, there are a lot of uncertain conditions. Fuzzy set model applied in this area can show status more clearly to the decision makers, how to make a good decision.

Secondly, fuzzy set model can work with traditional MRP very well. In nowadays, MRP engines are often in the core of the management and information exchange on the orders within SCM (supply chain management) (G. Daria). So, fuzzy MRP is a developed MRP system. 


\section{Niu and Dartnall}

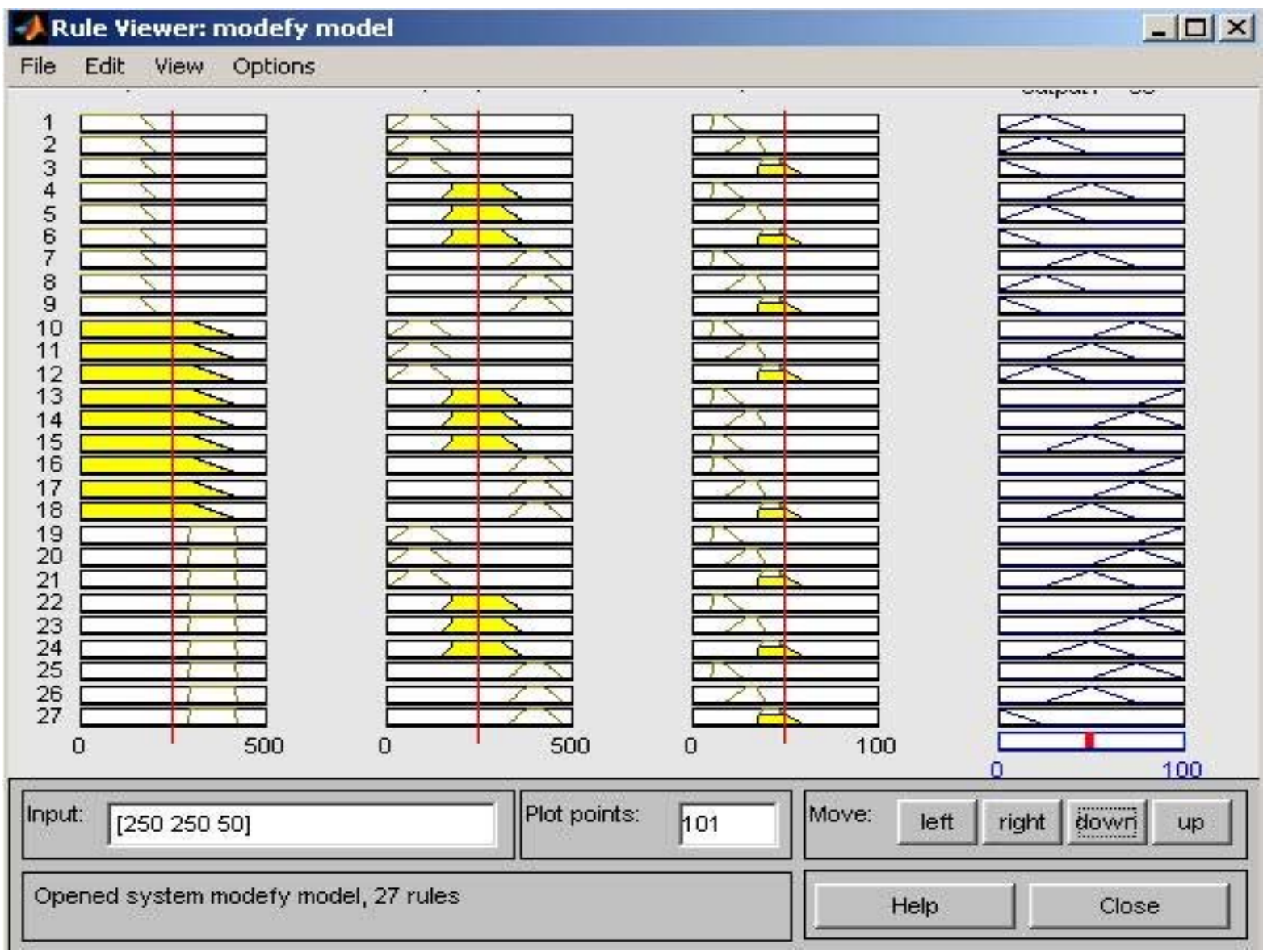

Figure 7: The result of Fuzzy-MRP 


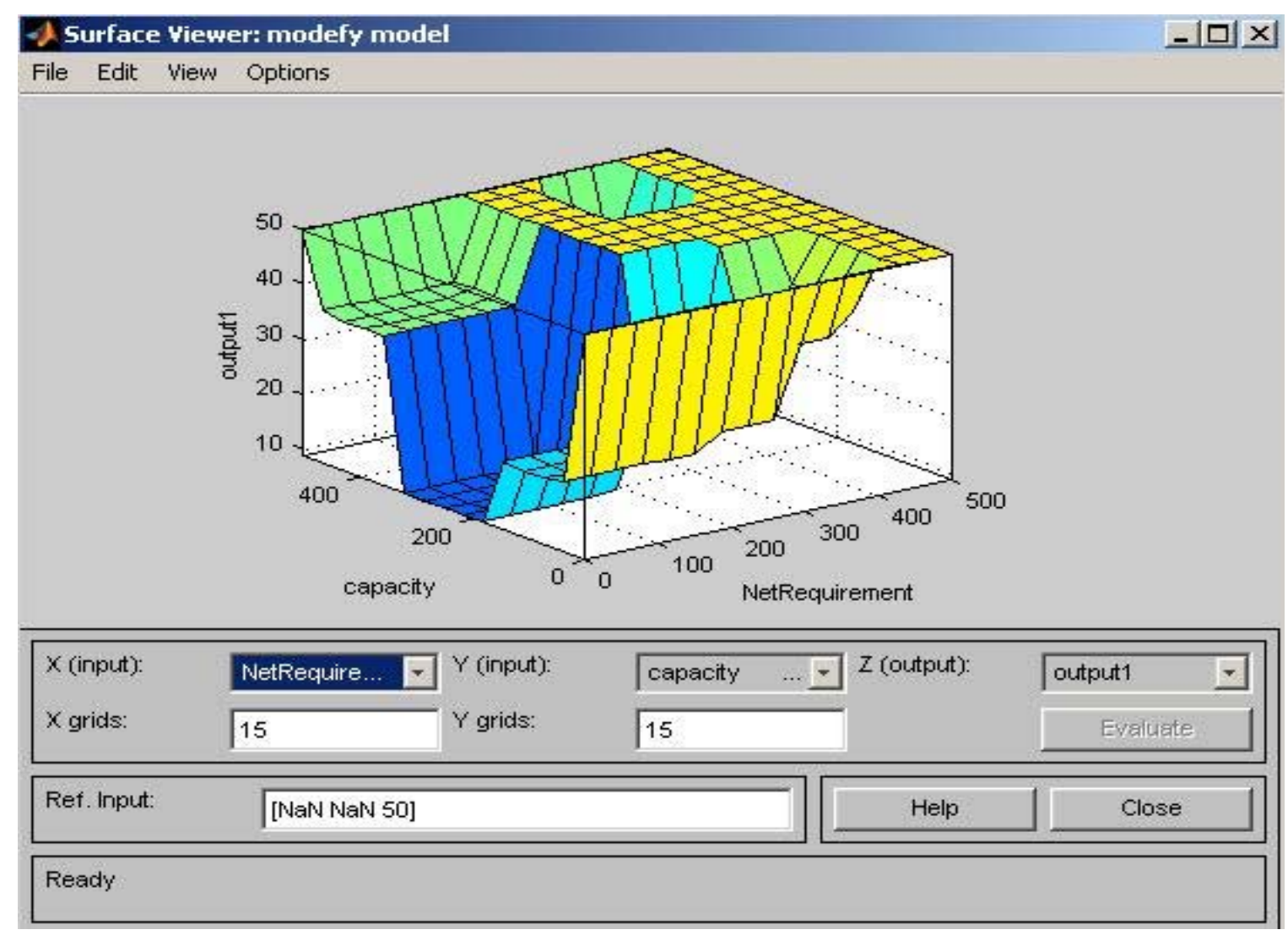

Figure 8: Result of Fuzzy-MRP

\section{REFERENCES}

Grabot, B., L. Geneste, G. R. Castillo and S. Verot. 2005. Integration of uncertain and imprecise orders in the MRP method. Intelligent Manufacturing 16:215-234.

Dubois, D., and H. Prade. 1989. Processing Fuzzy Temporal Knowledge. IEEE Transactions on systems Man and Cybernetics 19:729-744.

Efstathiou, J.. 1990. Applications of fuzzy set methodologies in industrial engineering. Fuzzy Sets and Systems 36:405-1532.

Daria, G., V. C. Machado. Using Fuzzy Logic to Model MRP Systems under Uncertainty.

Kahraman, C. 2007. Fuzzy set applications in industrial engineering. Information Sciences 177:1531-1532.

Orlicky, J., and G. Plossl. 1994. Orlicky's Material Requirement Planning. 2nd Edition, McGraw Hill Text.

Rondeau, L., R. Ruelas, L. Levrat, and M. Lamotte.
1997. A defuzzification method respecting the fuzzification. Fuzzy Sets and Systems 86: 311-320.

\section{AUTHOR BIOGRAPHIES}

JIPING NIU is a Master research student of University of Technology, Sydney. She received her BSME from Beijing Technology \& Business University.

W. JOHN DARTNALL is a Lecturer of University of Technology, Sydney. He received his ME from University of Technology, Sydney. 